\title{
Chemokine signaling controls endodermal migration during zebrafish gastrulation
}

\author{
Sreelaja Nair and Thomas F. Schilling * \\ Department of Developmental and Cell Biology University of California, Irvine 92697-2300 USA
}

\begin{abstract}
Directed cell movements during gastrulation establish the germ layers of the vertebrate embryo and coordinate their contributions to different tissues and organs. Anterior migration of mesoderm and endoderm has largely been interpreted to result from epiboly and convergent-extension movements that drive body elongation. We show that the chemokine cxcl12b and its receptor Cxcr4A restrict anterior migration of endoderm during zebrafish gastrulation, thereby coordinating its movements with mesoderm. Depletion of either gene product causes the endoderm to separate from mesoderm and migrate farther anterior than normal, due to disruption of integrin-dependent cell adhesion. This results in bilateral duplications of endodermal organs, which may have relevance to human organ defects.
\end{abstract}

\section{Keywords}

Danio rerio; chemokine; gastrulation; gastrointestinal; alimentary

A crucial feature of vertebrate embryogenesis is the coordinated morphogenesis of germ layers (endoderm, mesoderm and ectoderm) during gastrulation (1). Interactions between endoderm and mesoderm specify organ locations and symmetries (2). Defects in endoderm alter morphogenesis of mesodermal organs (e.g. heart, kidneys, and blood), whereas mesodermal defects disrupt the locations of the liver and pancreas (2-5). Morphogenesis is regulated by Wnt (6) and Nodal signaling (7) when cells are intermingled in a bipotential "mesendoderm" (8). However, relatively little is known about germ layer-specific pathways that establish organ rudiments. In zebrafish, mesendodermal organ progenitors involute at the gastrula margin (blastopore) and move anteriorly toward the animal pole (future head) while converging toward the midline (convergent-extension).

The chemokine receptor CXCR4 controls directional migration in many contexts and is expressed in endoderm. It is upregulated by endodermal determinants, Mixer and Sox $17 \beta$ (9-13) and is required for gastrointestinal vascularization (14). Of the two closely related zebrafish Cxcr4s, Cxcr4B regulates migration of many cell types (12,15-18), but no roles have been reported for cxcr4A during embryogenesis.

Zebrafish embryos deficient in Cxcr4A or cxcl12b, generated by injection with antisense morpholino oligonucleotides (MO), appeared morphologically normal (Fig. 1A-C; Fig. S1). However, analysis of $T g(g u t G F P)^{s 854}$ transgenic embryos in which the entire gut fluoresces (19) revealed duplications of endodermal organs at 56 hours post fertilization (hpf) (Fig. 1DF; Fig. S2) including the pancreas (normally on the right; Fig. S3A-F) and liver (normally on the left; Fig. S3A-C, G-I), a phenotype we call "viscera bifida" (cxcl12b-MO: 78\% n=23;

\footnotetext{
*Author for correspondence: e-mail: tschilli@uci.edu, phone: (949) 824-2479, fax: (949) 824-4709.
} 
cxcr $4 a$-MO 68\% n=37). At 26 hpf the intestine was also split bilaterally (Fig. 1G-I; 12bMO, $78 \% \mathrm{n}=18,4 a \mathrm{MO}, 76 \% \mathrm{n}=34$ ), as revealed by foxa 2 expression, whereas the floorplate of the neural tube was unaffected (Fig. 1H, I). No defects were detected in mesoderm or ectoderm (Fig. S3J-O; Fig. S4).

During gastrulation, $c x c l 12 b$ is expressed in mesoderm (Fig. 2A, C, E; Fig. S5A, B), whereas cxcr4a is expressed endoderm (Fig. 2B, D, F; Fig S5E, F) and both require Nodal signaling (Fig. S5C, D, G, H), suggesting that chemokine signaling regulates endoderm-mesoderm interactions. At the onset of gastrulation (6 hpf), sox32+ endodermal cells appeared normal in number but were displaced slightly anteriorly in $\operatorname{cxc} 12 b(83 \%, \mathrm{n}=82)$ and $\operatorname{cxcr} 4 a(74 \%, \mathrm{n}=62)$ morphants (Fig. 1J-L). Displacement became more pronounced by $8 \mathrm{hpf}$, as revealed by foxa 2 expression (Fig. $1 \mathrm{M}-\mathrm{O} ; 12 b \mathrm{MO} 88 \% \mathrm{n}=34,4 a \mathrm{MO} 85 \% \mathrm{n}=55$ ). In controls, the trailing edge of foxa2 + cells was 50-100 $\mu \mathrm{m}$ from the margin, whereas in morphants this gap was over 3 -fold larger [178-275 $\mu \mathrm{m}$ dorsally (D), 170-310 $\mu \mathrm{m}$ laterally (L), 186-340 $\mu \mathrm{m}$ ventrally (V)] (Fig. 1P). The leading edge was also displaced up to $100 \mu \mathrm{m}$, particularly ventrally. Displacement was not due to precocious endodermal internalization (Fig. S6), suggesting a later requirement for chemokine signaling in restricting endodermal movements anteriorly, toward the animal pole. We refer to this as endodermal "tethering".

Despite displacement of the endoderm, mesoderm was unaffected as assayed by no tail ( $n t l)$ at $6 \mathrm{hpf}$ (Fig. 1J-L), tbxl6 in paraxial mesoderm at $8 \mathrm{hpf}$ (Fig. S4A-C), and hand 2 in lateral plate mesoderm (LPM, Fig. S4D-F). Our results reveal an early distinction between endodermal and mesodermal cell behaviors before they separate from mesendoderm and implicate the cxc112b-Cxcr4A system as among the earliest known signals in endodermal morphogenesis.

If cxcl12b in mesoderm binds Cxcr4A in endodermal cells to restrict (tether) their movements, Cxcr4A should be required cell autonomously in the endoderm. To test this, we transplanted endoderm-targeted cxcr4a morphant cells into wild-type hosts (Fig. 2G-L). As an internal control, these cells (green-fluorescein) were co-transplanted with wild- type endoderm (redrhodamine) into the same locations (Fig. 2G, I, K) in unlabeled hosts at $4 \mathrm{hpf}$, and cell distributions were compared 4 hours later (Fig. $2 \mathrm{H}, \mathrm{J}, \mathrm{L}$ ). cxcr4a morphant endodermal cells moved, on average, $200 \mu \mathrm{m}$ further anteriorly than controls (Fig. 2M), demonstrating a cell autonomous requirement.

How does cxcl2b-Cxcr4A signaling regulate endodermal migration? Since $c x c l 12 b$ is maternally deposited and localized to mesoderm, from which endodermal cells separate during gastrulation, it seems unlikely to act as a chemoattractant here. However, increasing evidence suggests that chemokine signaling modulates extracellular matrix $(\mathrm{ECM})$ proteins [e.g. fibronectin (FN), laminin] and their receptors (integrins), which are required for gastrulation (20-24). FN in ECM binds secreted CXCL12 and presents it to CXCR4, causing its redistribution to leading edges of migrating cells (25). Of several $f n$ s in zebrafish, $f n l$ is expressed by mesoderm during gastrulation (26). Thus, FN in the mesodermal ECM might bind and present cxcl12b to $c x c r 4 a$-expressing endoderm, sensitizing it to chemokines. CXCR4 activation by CXCL12 also enhances integrin-dependent adhesion of renal carcinoma and small-cell lung cancer cells to FN $(22,27)$. Thus we considered both FN and integrins as potential downstream effectors during endodermal migration.

If this is correct, interfering with FN-integrin interactions should also disrupt endoderm migration. To test this, we treated gastrulating zebrafish embryos with RGD peptides (containing the tripeptide motif arginine-glycine-aspartate), which bind integrins and block signaling (28). This caused anterior displacement of endoderm at $8 \mathrm{hpf}$, similar to $c x c l 12 b$ and cxcr4a morphants (Fig. 3A, B; 40\%, n=52), and delayed convergence of endoderm towards 
the midline (Fig. S7A-F; 55\%, n=20). In contrast, convergence of LPM, which is required for gut morphogenesis (5), was unaffected (Fig. S7G, H) as in morphants (Fig. S4D-F). RGD peptide treatments of transgenic $T g(g u t G F P)^{s 854}$ embryos caused viscera bifida (Fig. 3C, D; $42 \% \mathrm{n}=65$ ) or situs inversus (Fig S7I, J; 14\% $\mathrm{n}=64$ ), even when applied at mid-late gastrula stages (viscera bifida, $32 \% \mathrm{n}=22$; situs inversus, $18 \% \mathrm{n}=22$ ), indicating that integrin-dependent interactions are essential throughout gastrulation.

These results argue against the presentation of FN-bound cxcl12b to Cxcr4A and instead suggest that chemokines control ECM-integrin-dependent adhesive interactions of endoderm. To test this, we assayed the ability of $c x c r 4 a$ morphant endodermal cells to adhere to FN-coated surfaces in in-vitro cell adhesion assays. A third fewer morphant cells remained attached when compared to controls, and adhesion was rescued by co-injection of integrin beta $1 \mathrm{~b}$ (itgblb) mRNA (Fig. 3E), confirming that chemokine signaling directly regulates adhesion of endoderm to FN.

Molecular interactions between CXCL12 and CXCR4 upregulate levels of integrin $\alpha$ and $\beta$ mRNAs in renal carcinoma cells to enhance their adhesion to FN (22). Thus, cxcl12b-Cxcr4A signaling in the endoderm may similarly regulate integrin levels. Of the integrin $\beta$ s expressed in zebrafish, itgblb is expressed maternally and ubiquitously during gastrulation (29).

Quantitative real time-PCR revealed a reduction in itgblb mRNA levels in whole embryos and in $c x c r 4 a$-MO endoderm (Fig. S7K), suggesting that the link between chemokines and integrins is, at least in part, a transcriptional one.

If endodermal defects in CXCL12b-CXCR4a morphants reflect disruption of ECM-integrin signaling, injecting itgblb mRNA into morphants should rescue these defects. itgblb mRNA injected into $c x c l 12 b$ and $c x c r 4 a$ morphants rescued intestinal bifurcations in a dose-dependent manner (Fig. 3F-K), either unilaterally (50-75 pg; Fig. 3I, J) or completely (100 pg; Fig. 3K; Table S1). Taken together, our results suggest that cxcl12b-Cxcr4A interactions promote integrin-mediated adhesion to tether endoderm to mesoderm during gastrulation.

Cell adhesion is a key regulator of gastrulation movements. E-cadherin mediates epiboly and anterior migration of prechordal mesoderm (30), integrin-alphaB allows mesendodermal cells to crawl on FN along the blastocoel roof (31), and fnl controls migration of myocardial progenitors towards the midline $(26,32)$ - a process that also requires another chemokine, apelin $(33,34)$. However, gut defects have generally been interpreted as secondary to defects in mesoderm migration. In contrast, our studies reveal an earlier requirement for ECM-integrin interactions directly in endodermal migration.

We show that endodermal migration towards the anterior is genetically separable from other gastrulation movements (35). We propose that chemokine-dependent expression of integrin tethers endoderm to mesoderm and that loss of this tether releases endoderm to move anteriorly (Fig. 4) secondarily causing viscera bifida because endodermal cells on either side, (presumably containing organ progenitors) (8), have farther to converge dorsally and do not reach the midline in time to fuse. Viscera bifida-like syndromes in humans, including intestinal cysts and ectopic pancreatic or liver tissue, are relatively common and not associated with spina bifida (ectoderm) (36), and defects in the CXCL12/CXCR4 signaling pathway may be an underlying cause.

For zebrafish endodermal cells, regulation of migration by controlling adhesion reconciles the recent observation that involuted endodermal cells initially move via a "random walk" rather than the directed migration displayed by mesoderm (37). Classically, chemokines are cytokines that induce chemotaxis in responding cells; CXCL12-CXCR4 interactions control homing of hematopoietic stem cells to the bone marrow, as well as migration of germ cells, neuronal progenitors and several metastatic cancers $(15-18,27)$. In some of these cases, however, there 
is evidence for a system more like the tether described here, where receptor-expressing cells are confined to a territory defined by ligand-expressing cells. Thus, chemokine-dependent changes in adhesion to the ECM may influence cell migration rates and directionality in many developmental and disease contexts.

\section{Supplementary Material}

Refer to Web version on PubMed Central for supplementary material.

\section{Acknowledgments}

We thank E. Raz for $c x c l 12 b$ and $c x c r 4 a$ constructs. We also thank our laboratory colleagues, especially S. Piloto for help with RT-PCR; and I. Blitz for reviewing the manuscript. Supported by NIH R01NS41353 and R01DE13828.

\section{REFERENCES}

1. Solnica-Krezel L. Curr Biol Mar;2005 15:R213. [PubMed: 15797016]

2. Roberts DJ. Dev Dyn Oct;2000 219:109. [PubMed: 11002332]

3. Alexander J, et al. Dev Biol Nov;1999 215:343. [PubMed: 10545242]

4. Biemar F, et al. Dev Biol Feb;2001 230:189. [PubMed: 11161572]

5. Ober EA, et al. Nature Aug;2006 442:688. [PubMed: 16799568]

6. Keller R. Science Dec;2002 298:1950. [PubMed: 12471247]

7. Schier AF. Annu Rev Cell Dev Biol 2003;19:589. [PubMed: 14570583]

8. Warga RM, Nusslein-Volhard C. Development Feb;1999 126:827. [PubMed: 9895329]

9. Chong SW, et al. Mech Dev Dec;2001 109:347. [PubMed: 11731248]

10. Dickinson K, et al. Dev Dyn Feb;2006 235:368. [PubMed: 16278889]

11. Fukui A, et al. Biochem Biophys Res Commun Mar;2007 354:472. [PubMed: 17239342]

12. McGrath KE, et al. Dev Biol Sep;1999 213:442. [PubMed: 10479460]

13. Yusuf F, et al. Anat Embryol (Berl) Aug;2005 210:35. [PubMed: 16047188]

14. Tachibana K, et al. Nature Jun;1998 393:591. [PubMed: 9634237]

15. David NB, et al. Proc Natl Acad Sci U S A Dec;2002 99:16297. [PubMed: 12444253]

16. Knaut H, et al. Neuron Sep;2005 47:653. [PubMed: 16129396]

17. Li Q, et al. J Neurosci Feb;2005 25:1711. [PubMed: 15716407]

18. Raz E. Curr Opin Cell Biol Apr;2004 16:169. [PubMed: 15196560]

19. Field HA, et al. Dev Biol Jan;2003 253:279. [PubMed: 12645931]

20. Hartmann TN, et al. Oncogene Jun;2005 24:4462. [PubMed: 15806155]

21. Howard JE, et al. Mech Dev Aug;1992 38:109. [PubMed: 1384652]

22. Jones J, et al. Exp Cell Res Nov;2007 313:4051. [PubMed: 17706641]

23. Watt FM, Hodivala KJ. Curr Biol Mar;1994 4:270. [PubMed: 7522915]

24. Winklbauer R, Keller RE. Dev Biol Aug;1996 177:413. [PubMed: 8806820]

25. Pelletier AJ, et al. Blood Oct;2000 96:2682. [PubMed: 11023498]

26. Trinh LA, Stainier DY. Dev Cell Mar;2004 6:371. [PubMed: 15030760]

27. Juarez J, Bendall L. Histol Histopathol Jan;2004 19:299. [PubMed: 14702198]

28. Ruoslahti E. Annu Rev Cell Dev Biol 1996;12:697. [PubMed: 8970741]

29. Mould AP, et al. BMC Cell Biol 2006;7:24. [PubMed: 16787535]

30. Montero JA, et al. Development Mar;2005 132:1187. [PubMed: 15703282]

31. Winklbauer R, et al. Int J Dev Biol Feb;1996 40:305. [PubMed: 8735942]

32. Sakaguchi T, et al. Development Oct;2006 133:4063. [PubMed: 17008449]

33. Scott IC, et al. Dev Cell Mar;2007 12:403. [PubMed: 17336906]

34. Zeng XX, et al. Dev Cell Mar;2007 12:391. [PubMed: 17336905]

35. Keller R, et al. Differentiation Apr;2003 71:171. [PubMed: 12694202] 
36. Stevenson, RE., et al. Human malformations and related anomalies. Oxford University Press; New York: 1993.

37. Pezeron G, et al. Curr. Biol Feb;2008 18:276. [PubMed: 18291651] 


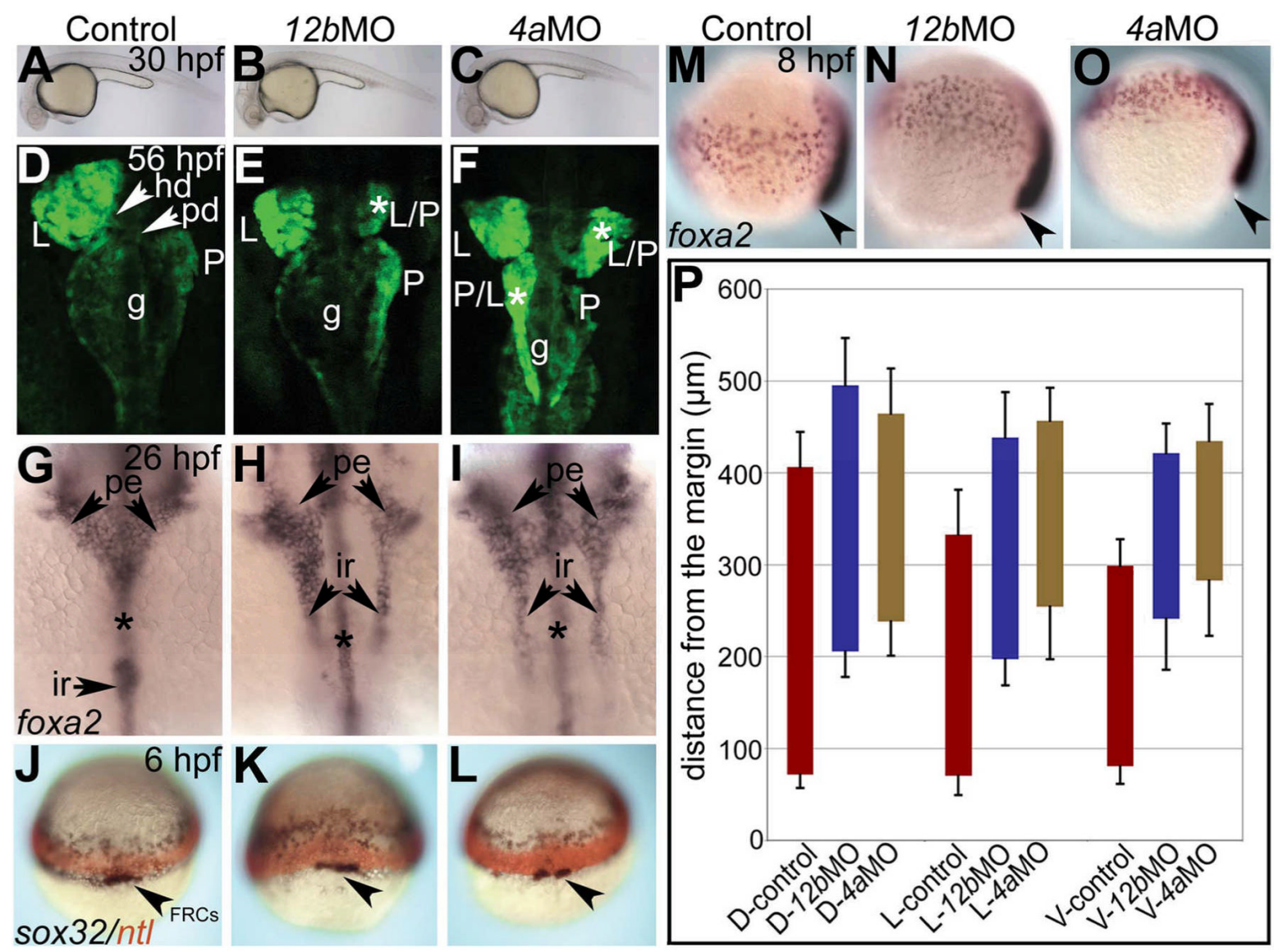

Figure 1. $c x c l 12 b$ and $c x c r 4 a$ are required for endodermal morphogenesis A-C, Bright-field images, live embryos, 30 hpf. Controls (A) cxcl12b (B) and cxcr $4 a(\mathbf{C})$ morphants; D-L, dorsal views, anterior to the top. D-F, $T g(g u t G F P)^{s 854}, 56 \mathrm{hpf}$, reveals duplicated liver (L) and pancreas (P) (asterisks) in confocal projections of $\operatorname{cxcl12b}(\mathbf{E})$ and cxcr4a (F) morphants. G-I, foxa2, $26 \mathrm{hpf}$, the intestinal rod (ir) bifurcates but not floor plate (asterisks). J-L, Double in situ for sox32 (endoderm-blue) and $n t l$ (mesoderm-red), 6 hpf. sox32 + cells, but not $n t l+$ or sox32+ forerunner cells (FRCs, arrowheads), move anteriorly (towards the animal pole and away from the margin). M-O, foxa $2,8 \mathrm{hpf}$, lateral views, morphant endoderm moves anteriorly (arrowheads indicate margin). P, quantitation of displacement of foxa2+ cells at dorsal (D), lateral ( $\mathrm{L}, 90^{\circ}$ from $\left.\mathrm{D}\right)$ and ventral (V, $180^{\circ}$ from $\left.\mathrm{D}\right)$ positions, 8 hpf. Controls (red bars), cxcl12b morphants (blue bars) and cxcr4a morphants (gold bars). g: gut, hd: hepatic duct, pd: pancreatic duct, pe: pharyngeal endoderm. 

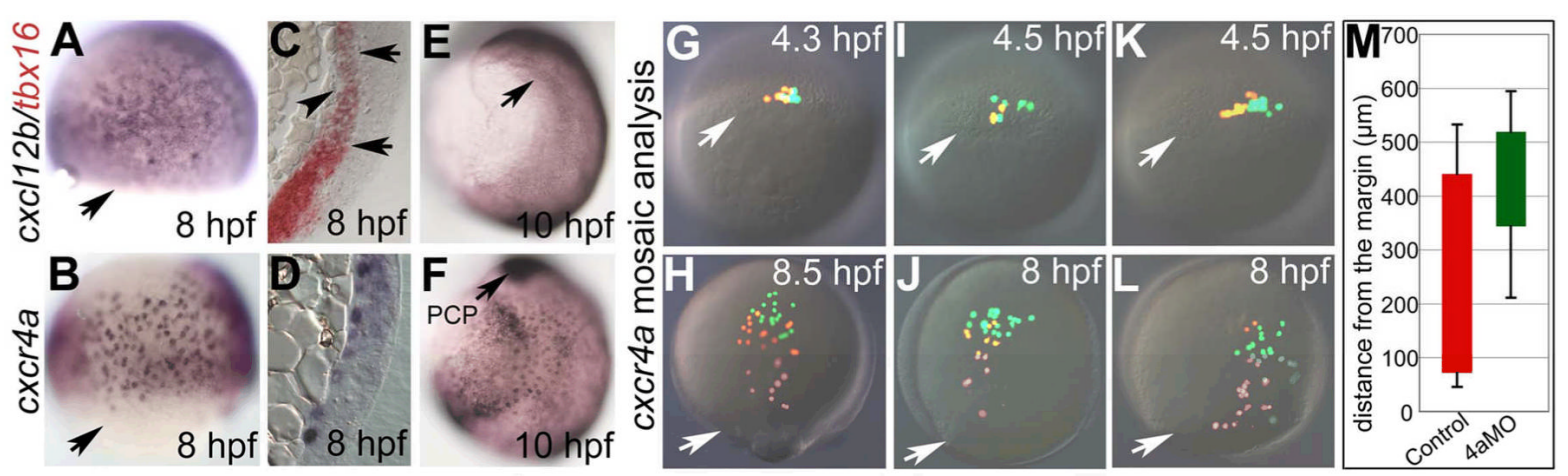

Figure 2. Mesoderm expresses $c x c l 12 b$ and endoderm requires $c x c r 4 a$ cell-autonomously

A-F, Whole-mount in situs, dorsal right (except C, D - transverse sections of gastrulae). (A) cxcl12b in mesoderm, $8 \mathrm{hpf}$, arrow indicates margin, (B) cxcr4a in endoderm. (C) Double in situ for $c x c l 12 b$ (blue) and $t b x 16$ (red), $8 \mathrm{hpf}$, confirms co-expression in region indicated by arrows, but not in endoderm (arrowhead). (D) Endodermal cxcr4a expression. (E) $c x c l 12 b$ in lateral mesoderm (arrow), $10 \mathrm{hpf}$. (F) cxcr4a in endoderm and prechordal plate (PCP, arrow), 10 hpf. G-L, cxcr $4 a$ morphant (green) and control cells (red) grafted into unlabeled hosts, lateral views at 4.3-4.5 (G, I, K) and 8-8.5 hpf $(\mathbf{H}, \mathbf{J}, \mathbf{L})$. M, Average leading and trailing positions of control (red bars) and cxcr $4 a$ morphant (green bars) transplanted cells ( $\mathrm{n}=7$ ). 

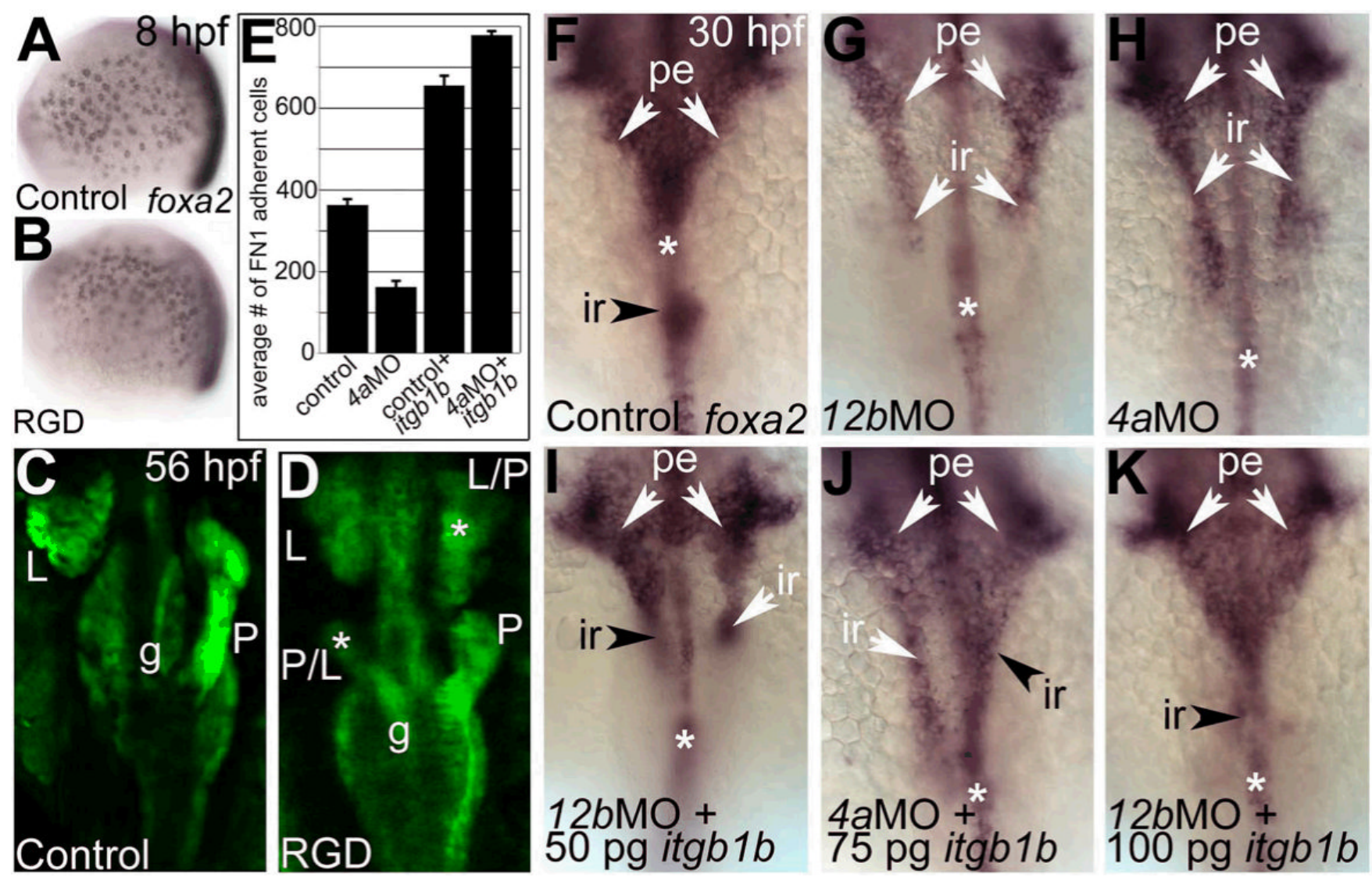

Figure 3. FN-integrin-mediated cell adhesion restricts anterior migration of endoderm A, B, Whole mount in situs for foxa $2,8 \mathrm{hpf}$, lateral view, dorsal (right). foxa2+ cells move anteriorly in RGD-treated embryos. C, D, Tg $(g u t G F P)^{s 854}, 56 \mathrm{hpf}$, Liver (L) and pancreas (P) duplications (asterisks) in RGD-treated embryos. E, reduced adhesion of cxcr4a morphant endoderm to a FN1-coated surface, and rescue by itgblb overexpression $(p=0.003$ and 0.0003 respectively). F-K, foxa2, $30 \mathrm{hpf}$. Dorsal views, anterior to the top, showing expression in pharyngeal endoderm (pe), floor plate (asterisk) and intestinal rod (ir, arrowhead), which bifurcates in morphants (white arrows). Injection of $50(\mathbf{I}), 75(\mathbf{J})$ and $100 \mathrm{pg}(\mathbf{K})$ of itgblb mRNA rescues the intestine partially (arrowheads - I and $\mathbf{J}$ ) or completely (arrowhead - K). 


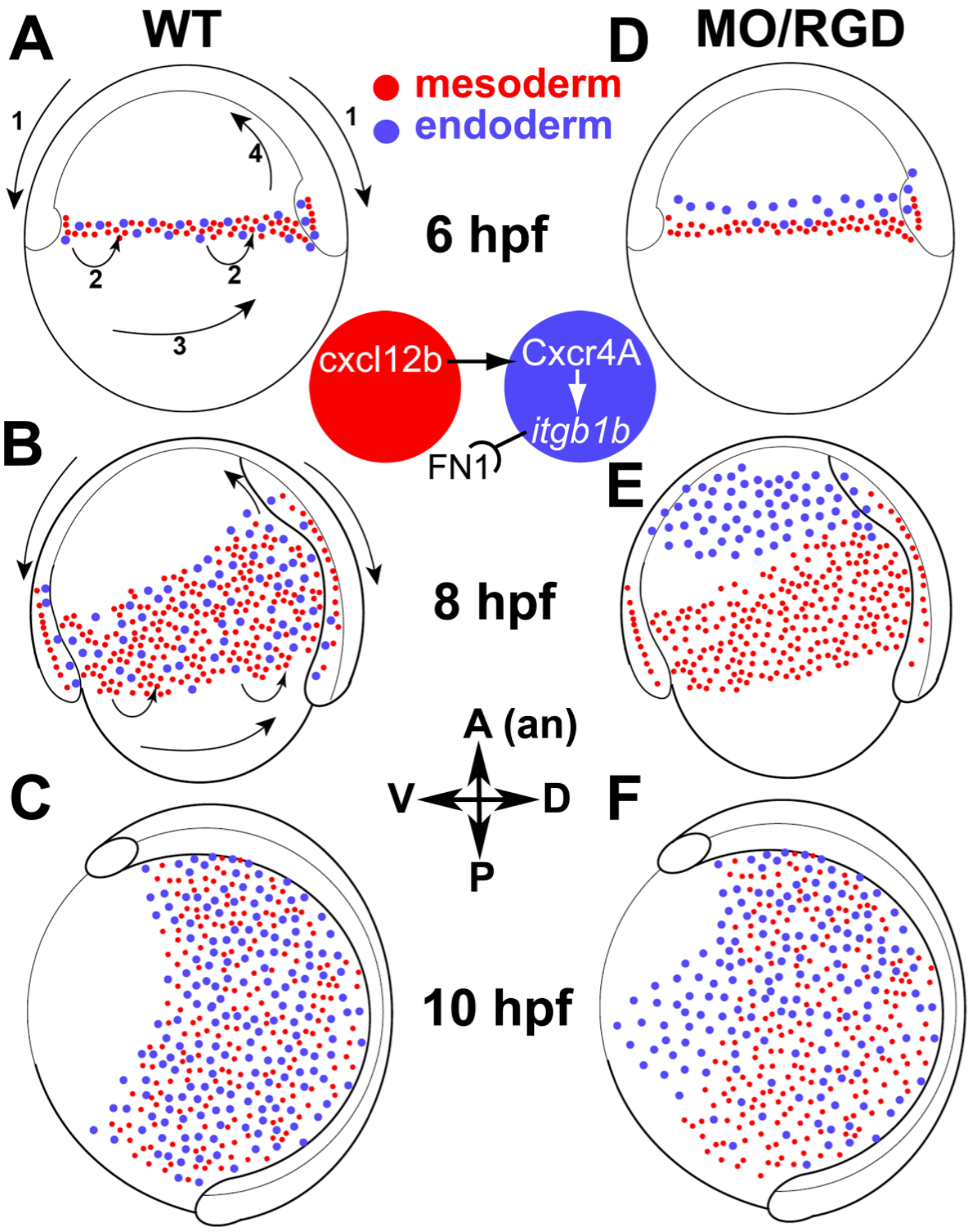

Figure 4. A chemokine-mediated tether model for endodermal morphogenesis

Diagrams of a wild type (WT) gastrula at 6,8 and $10 \mathrm{hpf}$, and $\operatorname{cxcl} 12 \mathrm{~b} / \mathrm{cxcr} 4 \mathrm{a}$ morphants or RGD-treated (MO/RGD) embryos, lateral view, animal pole (top), dorsal (right). . Endoderm (blue), mesoderm (red). Arrows: epiboly (1), involution (2), convergent-extension (3) and anterior migration (4). (A) At onset of gastrulation, cxcl12b+ mesoderm tethers cxcr $4 a+$ endoderm which coordinates mesendoderm migration $(\mathbf{B}, \mathbf{C})$ By the end of gastrulation midline convergence clears ventral cells. (D) In morphants or RGD-treated embryos, endoderm released from this tether migrates anteriorly. (E, F) Displaced endoderm in anterior and ventral positions does not reach the midline (ectopic ventral blue dots in $\mathbf{F}$ ), leading to organ duplications. Enlarged cells at center depict a molecular model in which cxcl12b from 
mesoderm signals through Cxcr4A in endoderm to upregulate itgblb expression, which binds FN1 in the mesodermal extracellular matrix. 